\title{
Superior warm-white light-emitting diodes integrated with quantum dot nanophosphors for high luminous efficacy and color rendering
}

\author{
Sedat Nizamoglu ${ }^{1}$, Talha Erdem ${ }^{1}$, Xiao Wei Sun ${ }^{2}$ and Hilmi Volkan Demir ${ }^{1,2}$ \\ ${ }^{1}$ Department of Electrical and Electronics Engineering, Department of Physics, and \\ UNAM - Institute of Materials Science and Nanotechnology, Bilkent University, Ankara, Turkey 06800 \\ ${ }^{2}$ School of Electrical and Electronic Engineering, Microelectronics Division; School of Physical and Mathematical Sciences, Physics and \\ Applied Physics Division, Nanyang Technological University, Singapore 639798 \\ Email:volkan@bilkent.edu.trandhvdemir@ntu.edu.sg
}

\begin{abstract}
Quantum dot nanophoshor hybridized warm-white LEDs are reported to exhibit high photometric performance of luminous efficacy exceeding $350 \mathrm{~lm} / \mathrm{W}_{\text {opt }}$ and color rendering index close to 90 at correlated color temperatures $<3000 \mathrm{~K}$.

(C)2011 Optical Society of America

OCIS codes: (130.0250) Optoelectronics; (160.4236) Nanomaterials
\end{abstract}

To date various theoretical investigations of white light-emitting diodes (WLEDs) have been conducted to realize efficient solid-state lighting (SSL) with a high color rendering index (CRI) approaching 90 and a high luminous efficacy of optical radiation (LER) above $350 \mathrm{~lm} / \mathrm{W}_{\text {opt }}[1,2]$. However, the reported experimental performances in terms of CRI and LER have been limited [3,4]. On the other hand, a CRI close to 90 is essential for a light source to reflect the true colors of illuminated objects, and an LER over $350 \mathrm{~lm} / \mathrm{W}_{\text {opt }}$ is important to provide an efficient spectral content matching the human eye sensitivity curve. As the current state-of-the-art, a warm-WLED with a LER of $274 \mathrm{~lm} / \mathrm{W}_{\text {opt }}$ and a CRI of 89 at a correlated color temperature (CCT) of $3100 \mathrm{~K}$ has previously been demonstrated by using nitride-based $\mathrm{Eu}^{2+}$ phosphors coated on a blue LED [3]. In this study, to outperform conventional phosphors photometrically, we analytically designed and experimentally demonstrated warm-WLEDs integrated with nanophosphors made of nanocrystal quantum dots (NQDs) on LED chips to achieve LER exceeding $350 \mathrm{~lm} / \mathrm{W}_{\mathrm{opt}}$ and $\mathrm{CRI}=89.2$ at $\mathrm{CCT}<3000 \mathrm{~K}[5]$.

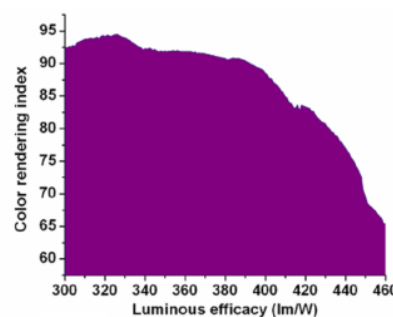

(a)

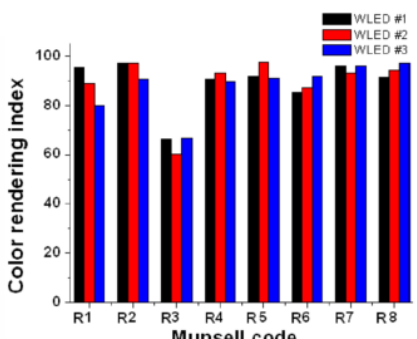

(b)

Figure 1. Color rendering indices vs. luminous efficacy of optical radiation (in $l \mathrm{~m} / W_{\text {opt }}$ ) for WLEDs integrated with NQD nanophosphors, and (b) color rendering indices of NQD-integrated warm-WLEDs (WLED \#1, \#2 and \#3) analyzed at different Munsell hues.

NQDs offer favorable properties for color conversion LEDs. They advantageously exhibit high photon conversion efficiency, stability and photobleaching thresholds [6]. Their optical properties can be conveniently adjusted by tuning size and shape, and using different material systems. Furthermore, their narrow emission linewidths enable the custom-design of a desired emission spectrum by using proper combinations of differently sized NQDs. Thus, NQDs are strong candidates for use in color conversion as an alternative to conventional phosphors. In operation, such WLEDs integrated with NQDs reply on the combined emission of the LED electroluminescence (as a result of electrically driving the LED) and the NQD photoluminescence (as a result of optically pumping the NQDs by the LED). However, since we use nanocrystals in a host medium (PMMApoly(methyl methacrylate)), the host effect and dipole-dipole interactions prevent us from exactly predicting the peak emission wavelength shifts, linewidth broadenings and relative emission strengths of different NQD components in the solid film. Thus, we need to perform numerical analyses and feasibility study to understand whether the intended high-quality white light generation can be achieved by using our green-, yellow- and redemitting CdSe/ZnS core/shell NQDs on blue LEDs, despite modification of their emission properties in the solid state. Our starting nanocrystals exhibit respective photoluminescence (PL) spectra peaking at 528, 560 and $609 \mathrm{~nm}$ in toluene, which are modeled as a Gaussian distribution for each color component. In our numerical simulations, by 
further varying the emission peak and strength of each color including the LED and NQDs over a total number of 1,180,980 cases, we investigated and evaluated feasible hybrid LED performance. The simulated data for the operating points of LER $>300 \mathrm{~lm} / \mathrm{W}_{\text {opt }}$ and CRI $>60$ are shown in Fig. 1(a) and these results reveal that our NQD nanophosphors on the blue LED are capable of achieving spectrally efficient and high-quality white light generation and that there is room to accommodate the changes in the optical properties of the NQD solids.

For the implementation of our first white light emitting diode (WLED \#1) design, we integrated $31.91 \mathrm{nmoles}$ of green-emitting NQDs, 1.42 nmoles of yellow-emitting NQDs, and 0.37 nmoles of orange-emitting NQDs on our blue LED. The orange-emitting nanocrystals allow for the color temperature to drop to obtain warm white shade and balance the luminescence of the green-emitting nanocrystals along with the blue LED. As a result, the chromaticity coordinates stay inside the white region while keeping LER and CRI high. Fig. 2(a) presents the emission spectra and chromaticity properties of this hybrid LED at different current injection levels at room temperature. The operating point at $12 \mathrm{~mA}$ corresponds to $(\mathrm{x}, \mathrm{y})=(0.425,0.378), \mathrm{LER}=357 \mathrm{~lm} / \mathrm{W}_{\mathrm{opt}}$, CRI $=89.2$, and $\mathrm{CCT}=2982 \mathrm{~K}$, which makes a warm-WLED at a desirably low CCT below $3000 \mathrm{~K}$ and satisfies at the same time the CRI demand for future SSL applications (with a CRI > 80). Moreover, this hybrid LED reaches an unprecedented LER level of $357 \mathrm{~lm} / \mathrm{W}_{\text {opt }}$. This is because these nanocrystal emitters enable us to reduce the deep-red emission $(\lambda>650 \mathrm{~nm})$, where the eye sensitivity quickly drops. We further investigated the device power conversion efficiency of our WLED to measure a power conversion efficiency of 0.11 , which corresponds to a luminous efficiency of approximately $40 \mathrm{~lm} / \mathrm{W}_{\text {electrical }}$. By using a state-of-the-art high-efficiency LED and NQDs, we predict that a luminous efficiency of $100 \mathrm{~lm} / \mathrm{W}_{\text {electrical }}$ and above is attainable.

For our second white light emitting diode (WLED \#2) design, we integrated 31.91 nmoles of green-emitting, 1.42 nmoles of yellow-emitting and 0.55 nmoles of orange-emitting NQDs. The emission spectra of this hybrid LED are presented in Fig. 2(b). The experimental operating point at $12 \mathrm{~mA}$ corresponds to $(\mathrm{x}, \mathrm{y})=(0.445,0.382)$, with LER=349 $\mathrm{lm} / \mathrm{W}_{\text {opt }}$, CRI=88.9, and CCT=2781 K. This second set of experimental results corresponds to a warmWLED with a further lower correlated color temperature because the optical emission intensity coming from orangeemitting nanocrystals is increased. Despite an accompanying minor decrease in CRI, a level of 88.9 is attained, again satisfying the need for CRI $>80$ and an LER of $349 \mathrm{~lm} / \mathrm{W}_{\text {opt }}$ is obtained, which is also higher than the prior reports of the best results for conventional phosphors [3]. In our last set of NQD integrated WLEDs (WLED \#3), we used 31.91 nmoles of green-emitting, 1.42 nmoles of yellow-emitting and 0.74 nmoles of orange-emitting NQDs to result in the emission spectra depicted in Fig. 2(c). The emission spectrum at $12 \mathrm{~mA}$ yields $(\mathrm{x}, \mathrm{y})=(0.452,0.376)$, with $\mathrm{LER}=339 \mathrm{~lm} / \mathrm{W}_{\text {opt }}, \mathrm{CRI}=87.8$, and CCT=2390 K, achieving a warm-WLED with high LER and high CRI.

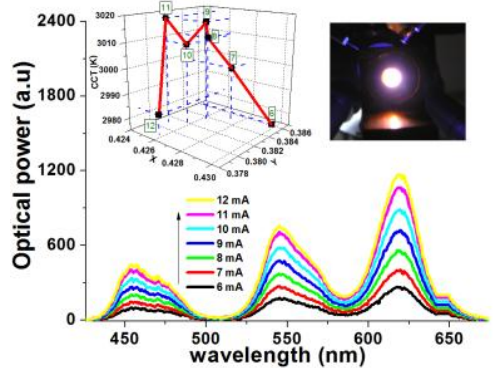

(a)

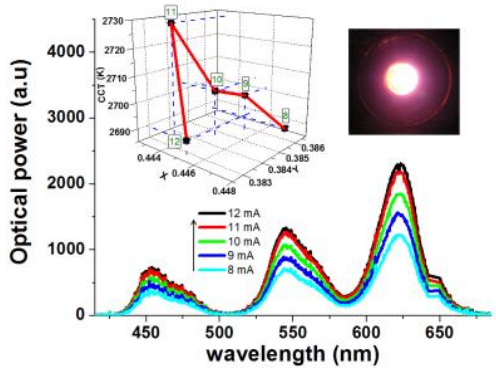

(b)

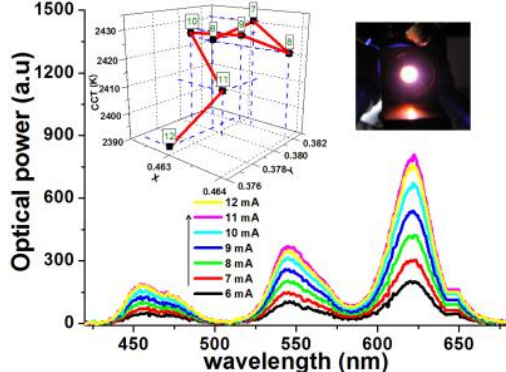

(c)

Figure 2. Electroluminescence spectra of (a) the first NQD-LED design (WLED \#1), (b) the second NQD-LED design and (c) the third NQDLED design integrated with green-, yellow- and orange-emitting CdSe/ZnS core/shell NQD nanophosphors on blue LED chips $\left(\lambda_{\mathrm{EL}}=452 \mathrm{~nm}\right)$ driven at different levels of current injection at room temperature, along with the corresponding (x,y) coordinates vs. correlated color temperature (CCT) and a picture of NQD-LED while generating white light. (In the inset, the values given in the squares represent the current injection levels at that operating point.)

To summarize, we designed and developed nanocrystal integrated warm-WLEDs with high luminous efficacy of optical radiation and high color rendering index at the same time. These hybrid LEDs hold great promise for future SSL applications.

We acknowledge financial support ESF-EURYI, EC FP7 N4E-NoE, TUBA- GEBIP, TUBITAK-BIDEB and TUBITAK 107E088, 109E002, 109E004, and 110E010, and NRF-RF-2009-09.

\section{References}

[1] J. M. Phillips et al., Laser \& Photon. Rev. 1, 307-333 (2007).

[2] A. Žukauskas, R. Vaicekauskas, F. Ivanauskas, R. Gaska, M. S. Shur, Appl. Phys. Lett. 80, 234-236 (2002).

[3] M. R. Krames, O. B. Shchekin, R. Mueller-Mach, G. O. Mueller, L. Zhou, G. Harbers, and M. G. Craford, J. Disp. Technol. 3, 2 (2007).

[4] S. Nizamoglu, G. Zengin, and H. V. Demir, Appl. Phys. Lett. 92, 031102 (2008).

[5] S. Nizamoglu, T. Erdem, X. W. Sun and H. V. Demir, Optics Letters 35, 3372-3374 (2010).

[6] S. V. Gaponenko, Introduction to Nanophotonics (Cambridge University Press, 2010). 